\title{
Biomechanical implications of rotation correction in orthodontics: Case series
}

\author{
Denny. J. Payyapilly ${ }^{1}$, Sunil Sunny ${ }^{2}$, Ayshakunja. A ${ }^{3}$, Anju Antony ${ }^{4, *}$ \\ ${ }^{1}$ Professor and HOD, ${ }^{2}$ Former Professor, ${ }^{3,4}$ Post Graduate Student, Dept. of Orthodontics, Annoor Dental College, Muvattupuzha, \\ Kerala, India
}

*Corresponding Author:

Email: anjuantony91@gmail.com

\begin{abstract}
According to Bacetti (1998) tooth rotation is defined as observable mesiolingual or distolingual intra-alveolar displacement of the tooth around its longitudinal axis. ${ }^{1}$ Tooth rotations cause difficulty in finishing fixed orthodontic treatment. In Begg's technique rotation springs are used to correct tooth rotations. These springs are engaged into the vertical slot of the bracket. In case of preadjusted edgewise technique and edgewise technique brackets doesn't have a vertical slot. So alternative methods are used to correct tooth rotations. ${ }^{2}$ Various methods include engaging NiTi arch wire into the bracket slot, off centering the bracket, using rotation wedges, creating a couple on the rotated tooth, by using ligature ties. ${ }^{3}$ The purpose of this case series is to discuss the biomechanics involved in rotation correction using bracket offsetting and couple.
\end{abstract}

Keywords: Couple, Bracket offsetting.

\section{Introduction}

According to Bacetti (1998) tooth rotation is defined as observable mesiolingual or distolingual intra-alveolar displacement of the tooth around its longitudinal axis ${ }^{1}$ many rotations are associated with an element of apical displacement and are difficult to correct. In the present case series, cases with rotated teeth, corrected using bracket offsetting and couple mechanics are presented along with the biomechanics.
Correction of Rotated Incisors with Bracket Offset

Case Report- 1: A 16 year old patient reported to the department of orthodontics with forwardly placed upper and lower front teeth. Patient had class II subdivision malocclusion with bimaxillary proclination on a class II skeletal jaw base. Patient had upper and lower anterior crowding (Fig. 1). Bonding was done and brackets on 12 and 22 were placed with distal offset. Rotation correction of 11 and 21 achieved within 3 months of time by engaging NiTi wire into the bracket (Fig. 2).
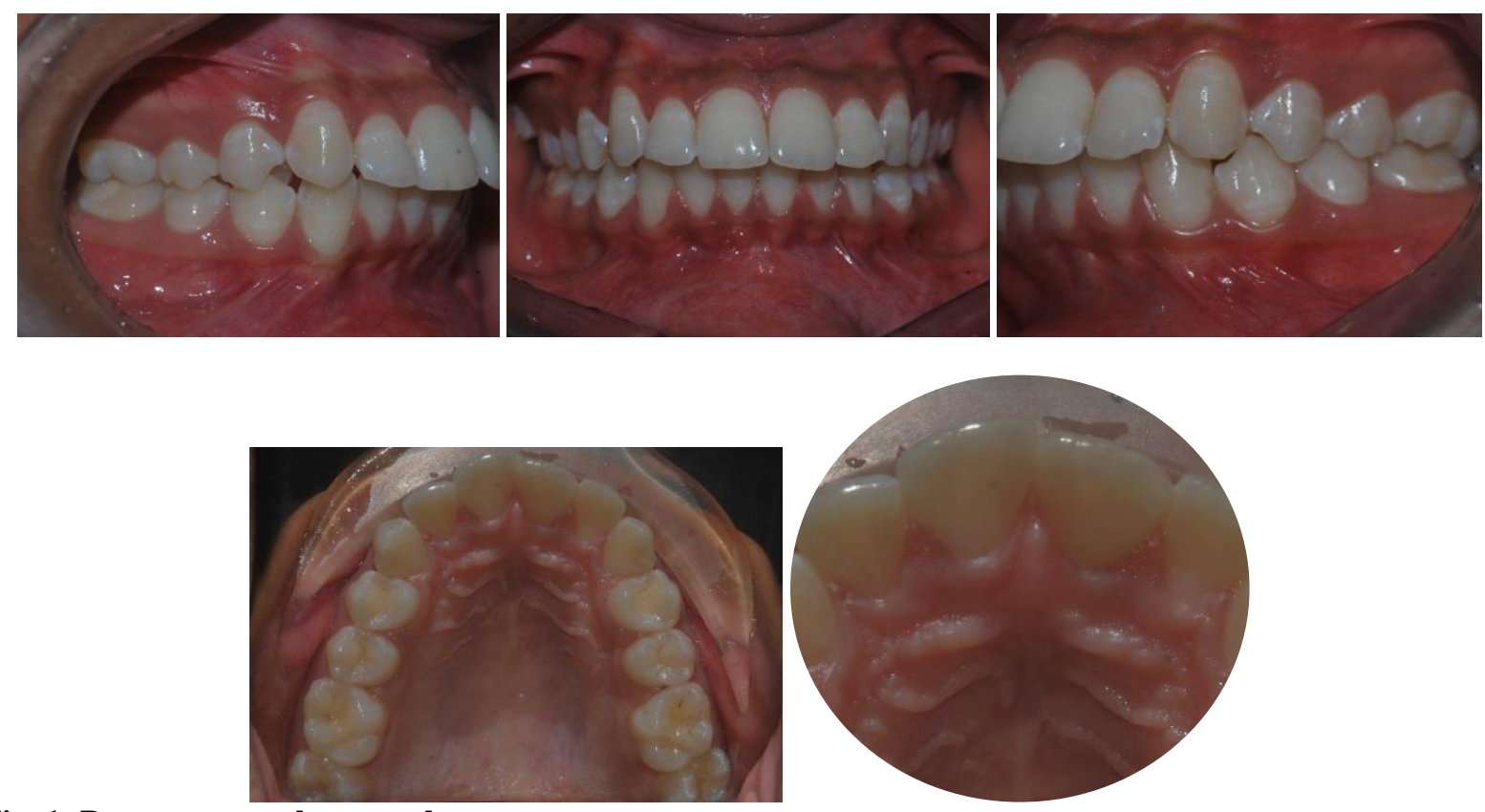

Fig. 1: Pretreatment photographs 

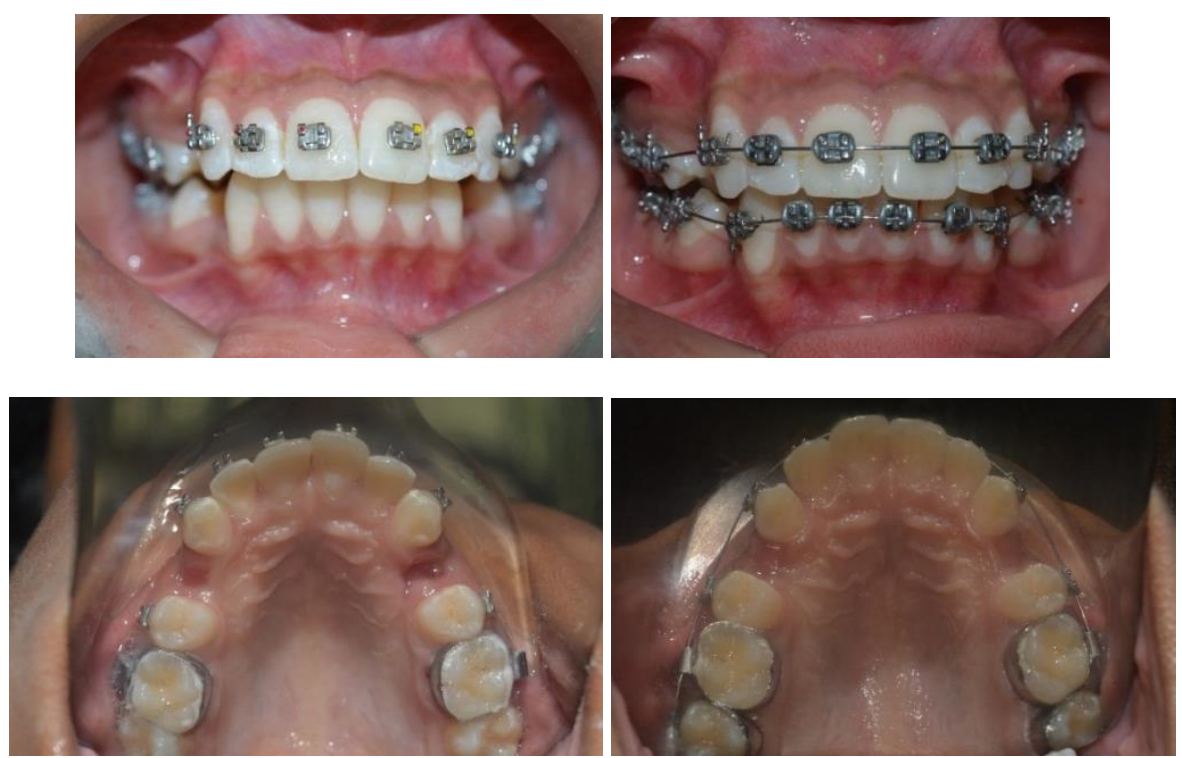

Fig. 2: Correction of rotated incisors with bracket offset

Rotation Correction with Couple Mechanics Case Report- 2: Twenty year old patient reported to the department of orthodontics with crowded upper and lower anteriors and forwardly paced lower jaw. Patient was diagnosed with super class I malocclusion bilaterally with upper and lower anterior crowding and cross bite in relation to 11 on a class III skeletal jaw base (Fig. 3). Patient had severely rotated 35 and 45. Open coil spring was placed passively in relation to 35 and 45 to prevent the distal movement of 33 and 43. A couple was applied on both 35 and 45. Derotation of 35 and 45 was completed within 3 months (Fig. 4).
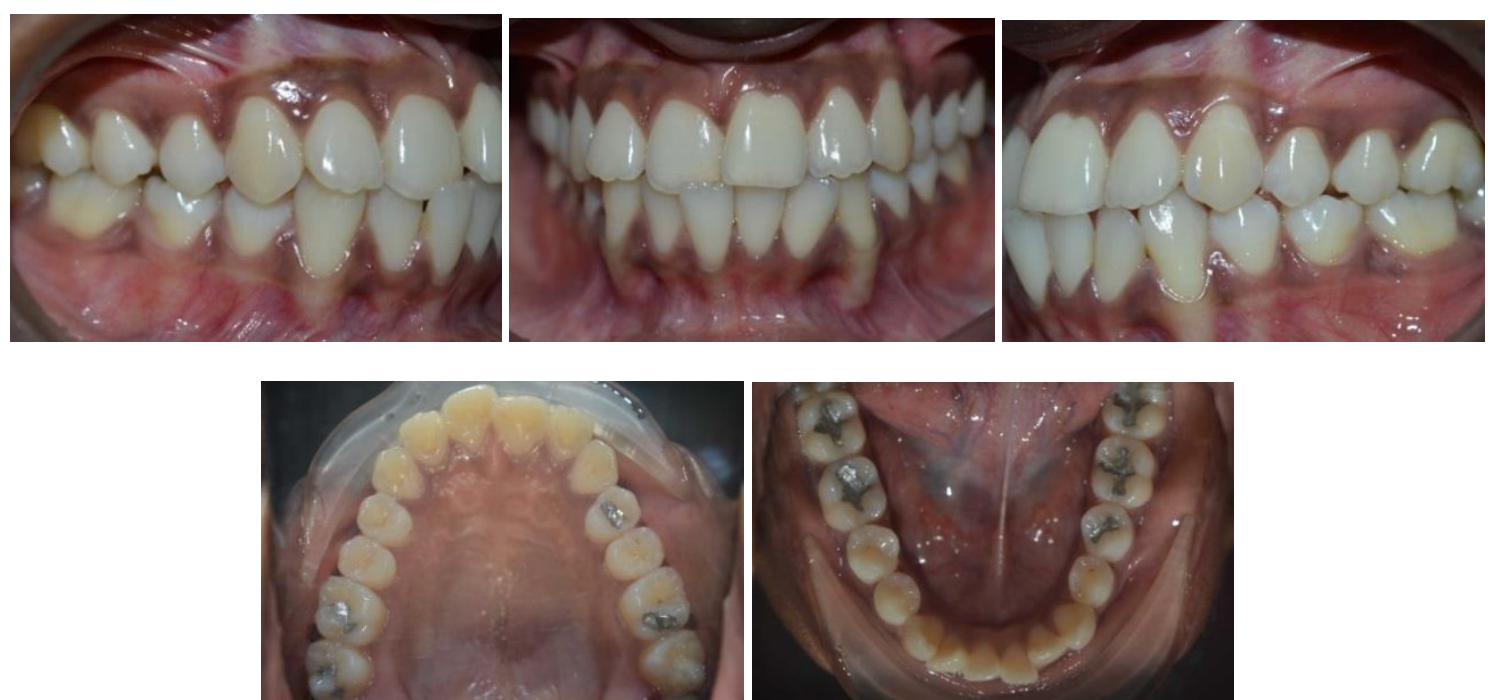

Fig. 3: Pretreatment photographs
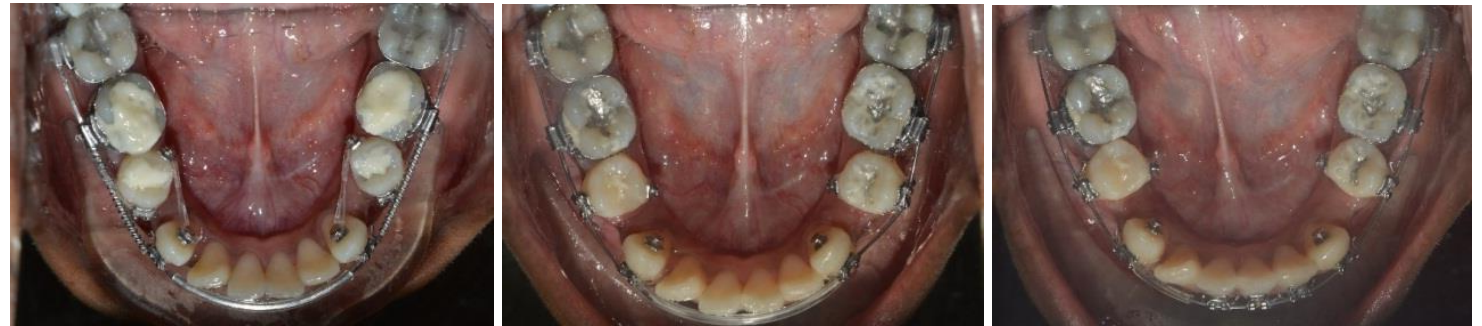

Fig. 4: Rotation correction with couple mechanics 
Rotation Correction with Couple and Sectional Niti Case Report- 3: A twenty five year old patient reported to the department of orthodontics with a chief complaint of forwardly placed upper front teeth and gap between the teeth. Patient was diagnosed with Angles class II malocclusion with open bite on a class II skeletal jaw base. The patient had 90 degree rotated and 35 and 45 in the lower jaw (Fig. 5). A couple was applied on 35 and 45, sectional NITI was also engaged on 35 and 45 along with couple. The derotation was complete within 4 months of time (Fig. 6).
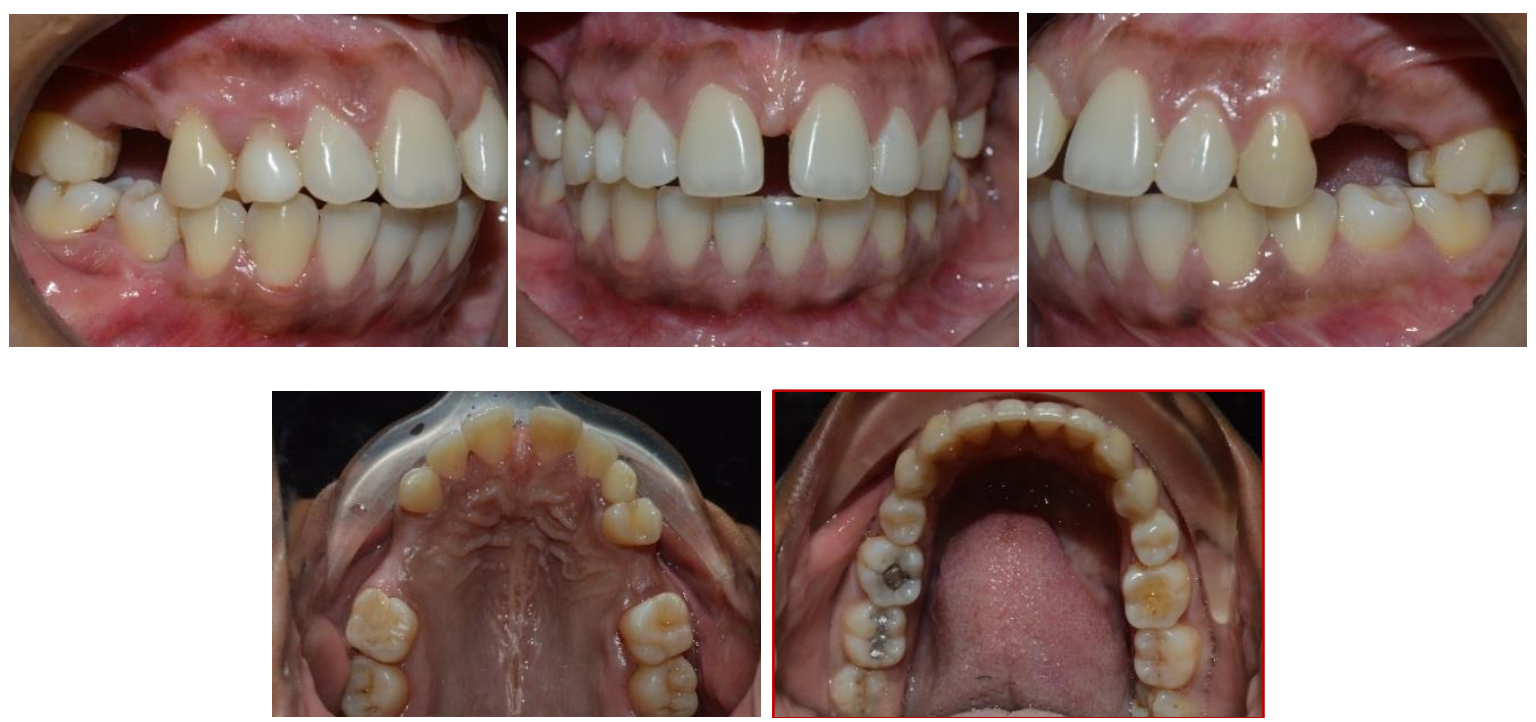

Fig. 5: Pretreatment photographs
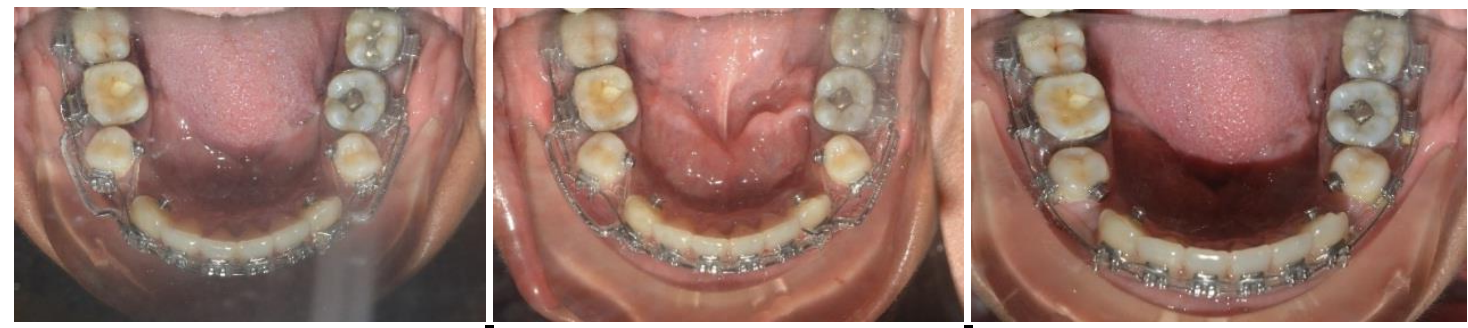

Fig. 6: Rotation correction with couple and sectional NiTi

\section{Discussion}

In the literature for the correction of rotated tooth, several treatment options have been proposed which including removable and fixed appliances, etc. Rotation control can be achieved without an additional ligature by using either twin brackets or single brackets with extension wings that contact the underside of the archwire to obtain necessary moment in the rotational plane of space. ${ }^{7}$ In fixed appliance, rotating springs were used in the Begg and Tip-Edge techniques, and most preadjusted and standard edgewise brackets do not had the vertical slots needed for such springs, and auxillary wire was used with such brackets for rotation correction. So alternative methods are used to correct tooth rotations. ${ }^{2}$ Various methods include engaging NiTi arch wire into the bracket slot, off centering the bracket, using rotation wedges, creating a couple on the rotated tooth, by using ligature ties the biomechanics behind the bracket offsetting is, if anterior teeth show rotations at the beginning of treatment, it is beneficial to place the bracket slightly in the direction of the rotation to aid in their correction. ${ }^{4} \mathrm{~A}$ force applied on a body, but not through the center of that body, results in translation and rotation ${ }^{5}$ and couple is defined as two forces of equal magnitude with parallel but non collinear lines of action and opposite senses. When a couple is applied to a body, the body will not translate but experience a pure rotation around its center of resistance irrespective of where the couple is applied. Equal and opposite force produce pure rotation. ${ }^{6}$ These are the simple techniques which are efficient for the rotation correction of anterior, posterior tooth single as well as multiple rotated tooth in either arch.

\section{Conclusion}

Tooth rotations can thus be corrected by offsetting the brackets, using NiTi arch wire and creating a couple on the rotated tooth. Derotated teeth have a strong tendency to relapse, for this reason it should be overcorrected if possible and retained full time for at least 6 months. 


\section{Reference}

1. Baccetti T. Tooth rotation associated with aplasia of nonadjacent teeth. The Angle Orthodontist. 1998 Oct;68(5):471-474.

2. Giancotti A, Cozza P, Romanini G. Correction of singletooth rotations with rotating springs. Journal of clinical orthodontics: JCO. 2000;34(11):656-8.

3. Hirpara N. Rapid Correction of Rotation With Modified Rotation Tie. Journal of Orthodontics \& Endodontics. 2015;1(2).

4. McLaughlin RP, Bennett JC. Finishing with the preadjusted orthodontic appliance. In Seminars in orthodontics 2003 Sep 1 (Vol. 9, No. 3, pp. 165-183). Elsevier.

5. Mulligan TF. Common sense mechanics. J Clin Orthod. $1980 \mathrm{Jul} ; 14: 180-9$.

6. Smith RJ, Burstone CJ. Mechanics of tooth movement. American Journal of Orthodontics. 1984 Apr 1;85(4):294-307.

7. Lohakare SS. Orthodontic removable appliance. Jaypee Brothers Medical Publishers Pvt Ltd; 2008. p. 47-48. 